\title{
REDES PERSONALES Y CAPITALES INSTITUCIONALES: LA REAL HACIENDA Y EL CABILDO DE QUITO A MEDIADOS DEL SIGLO XVIII
}

\author{
Tamar Herzog \\ Universidad de Chicago
}

Las relaciones entre redes personales y capitales institucionales, están en el centro de reflexión de este artículo. Tomando como ejemplo el papel de quien fuera tesorero de la Real Hacienda de Quito en las décadas de 1730 y 1740, quisiera argumentar a favor de la imposibilidad de entender el establecimiento de relaciones sociales, sin la mediación de las instituciones y viceversa. Por un lado, el éxito obtenido en un terreno, se reflejaba en la ampliación de posibilidades en el otro. Por otro, los individuos, que a veces se definían por un medio, en ocasiones distintas se identificaban con el otro, convirtiendo lo social y lo institucional en dos facetas del mismo problema, el de la constante reconfiguración del poder. 
Fernando García Aguado, aquel tesorero objeto de nuestro estudio, aparece en la documentación histórica quiteña como un núcleo aglutinador dentro de una amplia red social que incluía, ínter-alia, al presidente de la audiencia, al obispo de la ciudad, a un oidor y a muchos otros individuos pertenecientes a la élite local. ${ }^{1}$ En total, esta red abarcaba a unas 100 personas, todas padres de familia y, por tanto, todas representantes de un grupo mucho más extenso cuya existencia, aunque conocida, no se menciona expresamente. ${ }^{2}$ La ocasión por la que esta red fue descrita y, según unos, constituida, fue la pesquisa celebrada contra el presidente de la Audiencia José de Araujo y Río en la década de $1740 .^{3}$ Se trataba de un proceso iniciado por orden de Madrid que venía recibiendo quejas contra el mandatario desde 1736 . Por diferentes razones, la instrucción inicial de 1738 no se ejecutó, por lo que sólo una segunda orden datada de 1742 consiguió la apertura de la investigación, iniciada por fin en 1743. ${ }^{4}$ Esta demora suponía, entre otras cosas, la celebración de una pesquisa pre-anunciada. Tanto la parte que apoyaba al presidente, como la contraria, venían luchando desde hacía años a favor o en contra de la decisión del Consejo de Indias y ambas tuvieron mucho tiempo para formar y re-formular sus alegatos y ampliar y reforzar tanto sus alianzas como sus rivalidades.

\footnotetext{
La información consultada proviene de una multitud de documentos, la mayoría de los cuales se hallan en el Archivo General de Indias (en adelante A.G.I.) Escribanía de Cámara (en adelante F..C.) 914 (A-C), 915 (A-C) y 916 (A-C), 964; A.G.I. Quito 133, ff. 301-375; 134, pp. 71 ss.; 135 y 374 y del Archivo Nacional del Ecuador en Quito (en adelante A.N.Q.) Criminales 32, expediente 20.12.1743.

2 La red social mencionada, fue estudiada por mí en otra ocasión. Tamar Herzog, La administración como un fenómeno social: La justicia penal de la ciudad de Quilo (1650-1750), Madrid, Centro de Estudios Constitucionales, 1995, pp. 136 - 143.

${ }^{3}$ La biografía de José de Araujo y Río fue estudiada por M. Moreyra y Paz Soldán: "El limeño Don José de Araujo y Río presidente de la Audiencia de Quito y capitán general de Guatemala", Mercurio Peruano, Vol. 26, No. 250, 1945, pp. 506 - 529. Ver así mismo, lámar Herzog,Los ministros de ;a Audiencia de Quito ¡650-1750, Quito, Libri-Mundi, 1995, pp. 46 - 49.

${ }^{4}$ La pesquisa contra el presidente José de Araujo y Río fue estudiada por mí en otra ocasión. Tamar Herzog, Ritos de control, prácticas de negociación: Pesquisas, visitas y residencias y las relaciones entre Quito y /Madrid (1650-1750). Madrid, Fundación Hernando de I.arramcndi-Mapfre (en imprenta). La misma fue objeto, también, de varios artículos publicados por Luis Ramos Gómez, entre los que se pueden enumerar "Un ejemplo de la lucha por el poder en Quito". Cultura, Vol. 8. 1986, pp. 117 - 132; "La estructura social quiteña entre 1737 y 1745 según el proceso contra José de Araujo", Revista de Indias, Vol. 51, 1991, pp. 25 - 56; "La pugna por el poder local en Quito entre 1737 y 1745 según el proceso contra el Presidente de la Audiencia José de Araujo y Río", Revista complutense de historia de América, No. 18, 1992, pp. 179 - 196 y "El "bien común' como pretexto del Presidente José de Araujo para crear una compañía de soldados y prohibir el aguardiente de caña en Quito en 1737", Revista andina. Año 11, No. 2, 1993, pp. 381 - 401. Ver así mismo, las referencias a la misma en Kenneth J. Andrien: "Corruption, Self lnterest and The Political Culture of Eighteenth Century Quito" R. K. Matthews, ed.: Virtue. Corruption and Self Interest. Political Values in the Eighteenth Century. Bethlehem. Lchight University Press, 1994. pp. 270 - 296, en pp. 278 - 283 and in his The Kingdom of Quito 1690-1830: The State and Regional Development, Cambridge, Cambridge University Press, 1995, pp.173 179.
} 
La búsqueda de nuevos acercamientos y el fortalecimiento de redes sociales preexistentes ocurridos en Quito durante las décadas de 1730 y 1740, debe entenderse dentro de este ambiente de conflicto latente y, posiblemente, preeminente. Aunque la parte que apoyaba al presidente parecía más numerosa, frente a ella se presentó un grupo no menos importante, que incluía unos cincuenta padres de familia, entre los cuales se contaban tres comerciantes acaudalados y el fiscal de la Audiencia. Este grupo, además de sus amplias posibilidades económicas, tenía al parecer una cohesión interna bastante fuerte, estructurada en relaciones de familia, de comercio y de amistad.

Los documentos de archivo consultados no describen el medio por el cual los grupos se constituyeron. Aunque definen las relaciones entre los diferentes individuos que los componían en términos bastante claros, tales como la relación de familia, de amistad, de dependencia y de intereses comunes, no detallan la forma por la que estas relaciones fueron instituidas y la conexión entre unas y otras. Tampoco explican cuáles eran las ventajas de pertenecer a un conglomerado u otro, y cuáles habían sido las razones por las que unas personas, que podían pertenecer a ambos campos, o a ninguno, decidieron optar por uno de ellos. Sin embargo, y a pesar del silencio, creo que existen suficientes indicios que permiten valorar diversos aspectos de estas redes sociales según funcionaron en aquella ocasión, lugar y tiempo. Volviendo al propósito ya anunciado, el ejemplo escogido a este fin es la persona del tesorero de la Real Hacienda, Fernando García Aguado. ¿Qué era lo que aportaba éste a la red?, ¿Cuáles podían haber sido las razones que le motivaron a pertenecer a la misma?, ¿De qué manera aquella pertenencia influyó en sus actividades y en sus decisiones?. Sin pretender leer sus pensamientos y deseos, creo, sin embargo, que se dispone de algunos indicios para empezar a entender cómo se configuró su papel dentro de estructuras sociales más amplias y cómo aquello fue manipulado en beneficio propio.

Femando García Aguado era el tesorero de la Real Hacienda. Este cargo suponía cierto prestigio social y bastante control sobre la administración local, tanto la Audiencia -cuyos ministros recibían sus salarios y propinas de las cajas-, como el cabildo. Sin embargo, la forma más evidente por la que el tesorero podía influir en la vida institucional de Quito era mediante la venta y arrendamiento de oficios. A esta categoría de "oficios vendibles" pertenecían los cargos de alcalde provincial, regidor, alguacil, escribano y procurador, es decir, algunos de los cargos más 
importantes de la ciudad. ${ }^{5}$ El hecho de que fueran "vendibles" suponía que su ejercicio fuera delegado en una persona mediante un procedimiento público (llamado remate), seguido por una confirmación de los resultados del mismo -prácticamente de forma automática- por el Rey o los ministros reales según el caso. Este procedimiento tenía la forma siguiente: el oficio a rematar se declaraba "vacante" por la Audiencia. Un pregón avisaba al público de esta situación y llamaba a los interesados a presentar posturas para la compra del mismo. Mientras las ofertas llegaban a la Audiencia y la misma certificaba su recepción, los oficiales reales entraban en acción. Su primera misión era evaluar el oficio. Estudiaban las compras anteriores y decidían cual era el valor del oficio ahora ofrecido en venta. El precio fijado en esta instancia servía para examinar las posturas del público y decidir si eran suficientes o si, al contrario, hacía falta esperar otras ofertas. De modo informal, durante este período de espera, los oficiales negociaban con los candidatos e intentaban convencerles de mejorar sus ofertas. Al acabar esta fase, se procedía al remate público. En presencia de un ministro de la Audiencia y del tesorero de la Real Hacienda, el pregonero anunciaba públicamente cuál había sido la mejor oferta e invitaba a todos los oyentes a presentar contra-ofertas. A continuación, el ministro, a consulta del oficial real, anunciaba el nombre del ganador, quien debía afianzar su oferta inmediatamente.

Este procedimiento permitía la intervención de una gran variedad de agentes locales en el nombramiento de oficiales y de regidores. El tesorero, en particular, estaba bien ubicado para influir en los resultados del remate. Su influjo era especialmente notable en tres momentos distintos: al evaluar el precio del oficio, al asistir personalmente a la ceremonia del remate y al conferir con el oidor sobre la identidad del ganador y, en casos especiales, sobre la prórroga del término. Cada uno de estos instantes le permitía, si lo quería, manipular los resultados del proceso. Por ejemplo, el precio fijado podía ser intencionalmente alto, para asegurarse de que nadie quisiera comprar el cargo. El fracaso de la venta produciría dos consecuencias: el oficio quedaría vacante o podía rematarse en arrendamiento, en vez de compra. Un precio demasiado bajo, al contrallo, facilitaría la candidatura de ciertas personas. De la misma manera, la calificación de la mejor oferta se

\footnotetext{
La mejor fuente, todavía, para conocer las reglas de la venta de oficio es Antonio León Pinelo, Tratad: de confirmaciones reales de encomiendas, oficios y casos en que se requieren para las Indis: Occidentales, Madrid. 1630. Ver igualmente, Francisco Tomás y Valiente, La venta de oficios en India: (1493-1606). Madrid, Instituto Nacional de Administración Pública, 1982 [1972].
} 
daba fácilmente a la manipulación, ya que al considerar la misma se evaluaba no sólo la suma ofrecida sino también los términos de pago y el crédito de la persona (es decir, la posibilidad de que pagara lo prometido y a tiempo). Incluso la decisión de finalizar el remate, o de esperar a otras ofertas podía ser estratégica, ya que podría suponer el intento de excluir a algunas personas del concurso o, al contrario, la voluntad de garantizar la participación de otras. A veces, la misma identificación del oficio como "vacante" y el deseo de rematarlo provenía de los oficiales reales. En otras ocasiones, los mismos lograron convencer a las autoridades de que no era necesario vender el oficio, sino que desde el primer momento, se debía ofrecer en arrendamiento. Este era el caso más común en Quito durante el siglo XVIII a pesar de las cédulas reales que insistían en que era preciso vender (y no arrendar) los oficios. ${ }^{6}$ La diferencia entre venta y arrendamiento tenia amplias repercusiones, que no se limitaban al campo económico. El arrendamiento tenía, por naturaleza, un carácter temporal, con un alcance medio de tres años. Los arrendatarios, cuya asociación con las instituciones era de corto tiempo, solían tener menos experiencia en el oficio y su identificación con él era parcial. En vez de oficiales atados a la administración mediante su inversión económica en ella, el arrendamiento permitía vincular personas cuya identificación con el oficio era inferior y quienes, potencialmente por lo menos, podían sentirse más libres y, tal vez menos responsables que sus colegas. Además permitía el acceso de personas interesadas en influir en un determinado proceso judicial o en una decisión gubernamental inminente, las que, al acabar éstos, se desvinculaban de la administración.

Estas reglas y realidades, al parecer, se utilizaron por parte de Fernando García Aguado nuestro tesorero, con el fin de fortalecer una red social y hacerla más poderosa de lo que era anteriormente. Por las mismas circunstancias de la pesquisa contra el presidente Araujo y Río, este comportamiento del tesorero se puso de manifiesto durante la investigación. Por pertenecer a la red del presidente, su asociación con aquél lo expuso a la rivalidad del campo adverso, el cual se quejó contra la manera como García Aguado utilizó su oficio para, como lo calificó el campo contrario, "fines particulares". La posibilidad de censurar al tesorero también provenía del hecho de que el contador Joseph Suárez de Figueroa -su compañero

\footnotetext{
La situación en Quito durante el siglo XVIII y el recurso al arrendamiento se describen en Tamar Herzog. Mediación, archivos y ejercicio. Los escribanos de Quito (siglo XVII), Francfort, Vittorio Klostermann, 1996. pp. 77 - 95.
} 
en la administración de la hacienda real- se asoció al menos parcialmente con el grupo opositor, al cual estaba dispuesto a apoyar dando informes en contra del tesorero y calificando sus actuaciones mediante el uso de criterios de enemigo y no de colega. ${ }^{7}$

La intervención de Fernando García Aguado en la provisión de oficios en Quito, con el fin de constituir y apoyar una red social se inició, según denunciaron sus rivales, hacia 1734, es decir, incluso antes de la llegada del presidente José de Araujo y Río a Quito. En aquel año, la Audiencia decidió sacar a la venta ocho oficios de regidores. El tesorero se opuso a la medida alegando la necesidad de seguir la práctica anterior de arrendar los mismos. Gracias a su insistencia, pudo conseguir que la Audiencia cambiara su decisión y permitiera el remate en arrendamiento. En esta ocasión, al menos según alegaron posteriormente sus enemigos, García Aguado manipuló las ofertas y las sumas; por este medio, introdujo en el cabildo a parientes y allegados suyos. Su intención, decían sus rivales, era clara. Controlar el cabildo, al que su propio ingreso era prohibido mediante repetidas cédulas reales.

Aunque es imposible afirmar la veracidad de estas reclamaciones, es evidente que los nuevos arrendatarios, quienes llegaron a ocupar sus oficios en 1734 actuaron, en efecto, como un grupo cohesionado y de parecer común. Dada su importancia numérica en el seno del cabildo (ocho regidores), consiguieron convertir sus ideas en decisiones. Entre otras cosas, pudieron elegir en 1735 alcaldes ordinarios a su gusto, todos pertenecientes a la parentela del tesorero, lo que parece apoyar la versión de sus contrarios sobre la maquinación de los remates por aquél. ${ }^{8}$ Además, esta elección servía a los intereses particulares de Femando García Aguado, quien tenía procesos pendientes en el juzgado ordinario de la ciudad y quien, posiblemente, buscaba garantizar la obtención de resultados favorables a su cause

\footnotetext{
El conflicto entre el tesorero y el contador y la pertenencia de ambos a campos rivales se describe, por ejemplo, en la Real Cédula de pesquisa, fechada en Aranjuez el 22 de abril de 1742; en la petición id contador, sin fecha en: A.G.I., EC 914A, cuaderno 2, ff. 129 - 136 y en la carta del mismo a su protector en Lima (José Antonio de Santander), fechada en Quito el 19 de abril de 1737 en: A.G.I.. E 914A, cuaderno 3, ff.263 - 264.

8 A.G.I., EC 914A, cuaderno 1, ff. 152 - 153. Ver, así mismo, el "Manifiesto impreso que hace la ciudad de Quito sobre nulidades que intervinieron en las elecciones de los oficiales de justicia de 1735 A.G.I., EC 914B, cuaderno 8, ff. 104 - 125 y el certificado del escribano del cabildo Domingo López Urquta, fechado 2 de enero de 1735, A.G.I., EC 927, ff. 62 - 63.
} 
mediante la ubicación de jueces "convenientes". Según el grupo contrario, García Aguado volvió a entrometerse en los remates del año 1736, aunque esta vez con menos éxito. En esta ocasión, doce oficios fueron sacados a la venta. A pesar de sus insistencias -de que era preciso recurrir al arrendamiento y no a la venta- y por existir órdenes del virrey claramente favorables a ella, la Audiencia procedió a rematar seis regidurías en propiedad y sólo una en arrendamiento, quedando las demás vacantes. ${ }^{9}$ Como era de esperar y dentro de la lógica de aquella historia, los ahora compradores pertenecían al grupo opuesto al tesorero y se quejaban del control que éste ejercía sobre el cabildo. Aunque todos ellos insistían en que su postura y posterior compra del oficio era motivada solamente por el deseo de "hacer servicio a Vuestra Majestad y restituir su antiguo lustre a esta ciudad"10, era más que evidente que todos ellos querían limitar la influencia de García Aguado y, por extensión, la de su grupo, en el seno del cuerpo municipal. Aparte de identificarse como rivales del tesorero, la formación del grupo comprador en 1736 no era nada accidental. Según alegó uno de sus componentes en 1748, al encontrarse perjudicado por su pertenencia a esta formación, fue "reclutado" al cabildo por Lorenzo Nates y Lorenzo de la Madrid, dos opulentos mercaderes quienes le prometieron abonar el precio del oficio y hacerse cargo de cualquier obligación que pudiera resultar del ejercicio del mismo. ${ }^{11}$ Aparte de comprarle el oficio, le ofrecieron también ayuda adicional. Le prometieron llevarle a Cartagena y prestarle el dinero necesario para emplearse en actividades mercantiles. La necesidad de su adhesión le fue explicada en términos claros: él no tendría que hacer nada, ni tomar postura en favor de nadie. Ellos lo harían todo. Lo que buscaban, en realidad, eran personas honradas que pudieran "hacer personería" para ellos y sus intereses en el cabildo. Aunque ningún otro integrante del grupo confesó con la misma franqueza los motivos por los que optó a convertirse en regidor, hubo indicaciones de que el mismo método fue empleado también con José Herrera y Tomás Centeno del Villar, y tal vez con otros.

\footnotetext{
9 El virrey insistía en la necesidad de vender los oficios en propiedad y prohibía su arrendamiento. Carta al presidente de la Audiencia Dionisio Alcedo y Herrera, fechada en Lima el 13 de diciembre de 1736, A.G.L EC 915A, cuaderno 1, f. 125. Ver, igualmente, certificado del contador José Suárez de Figueroa de 2 de febrero de 1737, A.G.I., EC 915A, cuaderno 1. ff. 65 - 66.

${ }^{10}$ Carta de los siete regidores que compraron o arrendaron los oficios en 1736 al rey, fechada en Quito el 8 de marzo de 1737, A.G.L EC 915A. cuaderno 1. f. 48.

${ }^{11}$ A.N.Q., Notaría Primera 29. expediente del 27 de agosto de 1748. Ver, así mismo, su petición de Julio de 1748, A.G.I., EC 916A, pieza 14 y la sentencia de vista y revista de 8 de agosto de 1747 y 20 de noviembre de 1756, A.G.Í., EC 964.
} 
La comparación entre los regidores que accedieron a sus oficios en 1734 y los que lo hicieron en 1736, por lo tanto, revela la existencia de dos mecanismos distintos de manipular el sistema y garantizar el control del cabildo. Mientras la asociación creada en 1734 dependía de un poder de base institucional -la capacidad del tesorero para influir en los resultados de los remates-, la agrupación de 1736 -que incluía personas que no pudieron influir en el remate- contaba al contrario con un poder de base económica, de tipo adquisitivo.

La lucha entre las facciones seguía en 1737. Mientras García Aguado volvía a insistir en la necesidad de arrendar los oficios que quedaron vacantes el año anterior, el fiscal de la Audiencia Juan Valparda y la Ormasa -haciéndose portavoz del grupo contrario- criticó abiertamente esta postura. ${ }^{12}$ Dijo que "desde tiempo inmemorable" el tesorero, quien debería velar por los intereses del erario público, mantenía una práctica que les perjudicaba. En lugar de proteger los intereses regios, protegía los propios mediante la entrada en el cabildo de sus parientes y allegados. Por ser "materia que corre a su arbitrio" pudo conseguir su intento sin encontrarse con demasiada resistencia. Controlar $i$ los regidores, le permitía determinar la identidad de los alcaides ordinarios. AI final del día, tanto la administración como la justicia local dependían de él. La situación se degeneraba de tal manera, que incluso personas interesadas en entra: al cabildo acabaron desistiendo, por no querer enemistarse con el tesorero, ahor; percibido como un individuo poderosísimo quien, además, estaba a la cabeza de un grupo social importantísimo.

De un modo u otro, la intervención de Valparda y la Ormasa en lo ocurrí c. en el cabildo en 1737, marcó la entrada de nuevos elementos en el escenario, ya que en la misma época el nuevo presidente de la Audiencia, José de Araujc y Río y posiblemente uno de los oidores, se integraron al bando contrario. La identificación del presidente con el grupo liderado por García Aguado se puso inmediatamente de manifiesto, al menos según sus rivales, cuande decidió apoyar los intereses de aquél y, en lugar de vender o arrendar los oficios, nombró a su gusto cinco regidores interinos. De este modo, y esquivando los procedimientos ordinarios de remate, pudo evitar que el poder económico de*

\footnotetext{
12 Carta del fiscal de la Audiencia de Quito, Juan Valparda y la Ormasa al rey, fechada en Quito el 1 marzo de 1737, A.G.I., EC 914A, cuaderno 1, ff. 31 - 34.
} 
grupo contrario al tesorero le pudiera ganar las regidurías, como ocurrió en 1736. Además, haciendo causa común con García Aguado, el presidente instruyó a los regidores nombrados por él para votar por alcaldes ordinarios a dos yernos del tesorero. Fracasado su intento, por no conseguir el apoyo de la mayoría de los cabildantes, Araujo y Río confirmó la voz de la minoría -la de los cinco regidores nombrados por él- y ordenó al cabildo aceptar a los yernos del tesorero como alcaldes legítimos. El presidente, por lo tanto, utilizó las prerrogativas de su oficio para conseguir lo que de otra manera era imposible, dada la creciente importancia numérica del grupo rival y la amplitud de sus recursos económicos. Adoptó la misma táctica en los años posteriores, interviniendo tanto en el nombramiento de regidores, como en la elección de alcaldes. En 1738, al parecer, con vistas a las elecciones de 1739, el presidente convenció a algunos de sus allegados (Tomás Pérez Guerrero, Francisco Javier Larrea, Bernardo de León y Ramón Juachin Maldonado) a presentar su candidatura a las regidurías vacantes. ${ }^{13}$

La formación de redes destinadas a controlar las instituciones, y la posibilidad que tenían algunos funcionarios de proporcionar a dichas redes los mecanismos requeridos a fin de acceder a diversas instancias, lo que a su vez permitía al grupo ganar nuevas adhesiones, también se pusieron de manifiesto en Quito en las elecciones de los alcaldes ordinarios. De nuevo, esto resulta evidente al analizar el papel de García Aguado. Aparte de las épocas en las que el tesorero controlaba la voluntad de la mayoría de los cabildantes y, por lo tanto, podía conseguir la elección de quien quisiera, García Aguado también podía manipular las elecciones por otros medios. Por ejemplo, dentro de las funciones de su oficio se hallaba la obligación de asegurar que los deudores de la Real Hacienda no tuvieran voto en el cabildo hasta pagar lo adeudado. La identificación de los deudores dependía de los oficiales reales y, a pesar de parecer simple, la materia era altamente conflictiva. Los privados de voto, apoyados por los suyos, intentaban probar el buen estado de sus cuentas con el fisco, y los del grupo opuesto se esforzaban en constatar lo contrario. La respuesta a la pregunta de quiénes eran los deudores, además, no era sencilla, ya que dependía del acceso a los libros de la caja y de la necesidad, a veces, de remontarse muchos años atrás. Además, a menudo se

13 Esta era la versión de Simón Álvarez Monteserín, quien fue miembro del grupo rival. Ver su petición sin fecha. A.G.I., EC 914A, cuaderno 2, f. 153 y 915A, cuaderno 4, f. 246. 
discutía cuándo era el momento fijado para la paga de ciertas sumas, y si la responsabilidad era de unos o de otros. En Quito, por lo menos, la identificación de los deudores se convirtió en un tema clave en las décadas de 1730 y 1740.4

Relacionada tal vez con lo que algunos autores identificaron como una crisis económica acaecida en la ciudad en aquella época, la cuestión, sin embargo, no dependía sólo de la existencia de deudas, sino de la manera en que ésta fue utilizada dentro de un clima de crispación y de existencia de bandos rivales en el cabildo y fuera de él. En 1736, por ejemplo, el alcalde provincial y el regidor decano apelaron ante el virrey la decisión de excluir a varios regidores del derecho al voto, alegando que las listas preparadas por los oficiales eran falsas. ${ }^{15}$ Los oficiales reales de por sí y a raíz de sus enfrentamientos particulares el uno con el otro, participaron en la discusión y se contradijeron entre sí. En 1737, el tesorero Fernando García Aguado determinó que nueve de los cabildantes eran deudores. ${ }^{16} \mathrm{El}$ contador, al contrario, afirmó que sólo se trataba de 5 de ellos, ya que el sexto pagó todas sus deudas y el séptimo sólo debía 40 pesos, los cuales no le habían sido cobrados por un error de los oficiales reales y no por negligencia suya. ${ }^{17} \mathrm{La}$ postura de cada uno de ellos producía otro balance de fuerzas en el cabildo j otorgaba, alternativamente, el poder a un grupo u otro. Mientras el tesorero mantenía su apoyo a las personas de su red, el contador parecía ahora apoyar los intereses contrarios. La importancia de la discusión era tal, y su resultado tan incierto, que es posible que el presidente de la Audiencia acabara prefiriendo ignorarla del todo. Según la versión de sus rivales, Araujo y Río omitió intencionalmente referirse a los informes de los oficiales en la Audiencia. Pidiendo a los oidores confirmar la voz de la minoría en la elecciones de 1737 según y

14 El análisis a continuación se basa en el estudio de las actas de las elecciones en el seno del cabildoquiteño, según los libros de cabildo de aquellos años, lodos localizados en el Archivo Municipal de Quito

15 El alcalde provincial y el regidor decano tuvieron que apelar a Lima porque el presidente y la Audiencia se negaron a oír sus quejas. Representación del procurador José Villanueva ante la Audiencia de Lima, en nombre de José Sánchez de Orellana y José Hidalgo Pinto, A.G.I.. 915A, cuaderno 4, ff. 220 - 236.

16 Certificado del tesorero Fernando García Aguado del 23 de marzo de 1737, A.G.I., EC 914B, cuadeno: 5, ff. 118 - 119. Se trataba de las personas siguientes: Simón Álvarez Monteserín, José Herrera, Juan José Sánchez de Orellana, Joachin Gómez Laso de la Vega, Tomás Pérez Guerrero, Juan Poncc de León (Conde de Selva Florida) y Antonio Pastrana.

17 Certificado del contador José Suárez de Figueroa, A.G.I., EC 914B, cuaderno 5, ff. 120 - 121. La discusión entre el contador y el tesorero duró algún tiempo. Ver los documentos insertos en 1T. 12 $-128$. 
como le convenía, no dijo nada sobre la existencia de aquel informe y de su contenido. ${ }^{18}$ En consecuencia, los oidores pensaron que la voz de la mayoría contaba con 7 regidores y la voz de la minoría con 5 . Sin embargo, considerando la información contenida en el informe de los oficiales, el campo apoyado por el presidente sólo contaba con 3 votos, ya que dos de sus integrantes eran deudores, mientras que el campo opositor seguía con 7. La minoría, que antes parecía casi accidental, por depender de un solo voto (la diferencia entre 5:7 y 6:6), era en realidad menos sólida que nunca, por representar una relación de 3:7. El presidente, al parecer, tomó la misma actitud el año posterior cuando, utilizando su autoridad personal e institucional, prohibía el acceso del escribano Diego Arias Altamirano a la sala del cabildo, por lo que el decreto que incluía los nombres de los deudores no fue leído a tiempo y antes de la votación. Este hecho garantizó que tres deudores pertenecientes al campo del presidente y del tesorero votaran a pesar de la prohibición legal. ${ }^{19}$ El informe, al contrario, no perjudicó a ninguno de los integrantes del otro grupo. Este resultado se podía esperar, ya que el informe fue elaborado por el contador Suárez Figueroa quien por aquél entonces ya se consideraba aliado del campo adverso al presidente. Además, el mismo fue materialmente preparado la noche anterior, en la casa de Lorenzo Nates, un miembro activo de aquel grupo. ${ }^{20}$ La lucha por la identificación de los deudores se intensificó de tal manera, que en 1739 los informes de los oficiales reales ya no bastaban y cada grupo, utilizando sus contactos con la Audiencia, intentaba afirmar que este tribunal y no los oficiales tenía la última palabra al respecto. Mientras el fiscal Valparda y la Ormaza pedía la nulidad de las elecciones por calificar a algunos de los votantes como deudores, el presidente, a su vez, prohibía al cabildo elegir a Antonio Pastrana por considerarle deudor. ${ }^{21}$

${ }^{18}$ Carta ya citada del fiscal Valparda y la Ormasa al Consejo de Indias, fechada 1 de marzo de 1737. Ver, así mismo, el certificado del contador José Suárez de Figueroa del 2 de febrero de 1737, A.G.I., EC 9I4A, cuaderno 1, ff. 68 - 71.

${ }^{19}$ Ver. por ejemplo, la petición ya citada de Simón Álvarez Monteserín. Araujo y Río negó esta versión. Alegó no haber sabido que el escribano llevaba el decreto de los oficiales reales y haber pensado que se hallaba en las proximidades del cabildo por otras razones. Ver su versión, A.G.I.,EC 914A, cuaderno 4, ff 125 - 189, en f. 166 y en su carta fechada en Quito el 18 de julio de 1739, A.G.LEC 914C, f. 45.

20 Testimonio del escribano Diego Arias Altamirano del 5 de agosto de 1743, A.G.I., EC 914A, cuaderno 2, ff. 115 - 118. Ver, así mismo, el proceso formado por el presidente contra Diego Arias Altamirano, A.G.I.. EC 9I4B, cuaderno 5. ff. 237 - 251.

${ }^{21}$ Petición del fiscal Valparda y la Ormasa del 7 de enero de 1739 y certificado del escribano de cabildo Domingo López Urquía del 5 de enero de 1739, ambas en A.N.Q., Oficios 29: expediente de 10 de enero de 1739. 


\section{Conclusiones}

En perspectiva histórica, una de las quejas más persistentes contra el presidente José de Araujo y Río, era su decisión de no quedarse al margen de los diferentes campos y grupos enfrentados en Quito. En su capacidad de primer mandatario, su inserción en un bando suponía la virtual muerte política del otro. De pronto, los miembros del grupo desfavorecido se vieron excluidos de la vida pública: no pudieron ostentar cargos -incluso cuando éstos dependían del cabildo-, ni podían acceder a comisiones, ni defender sus intereses. Como era de esperarse, poco tiempo después, los excluidos se convirtieron en sus peores enemigos, denunciando sus actividades a Madrid y, posteriormente, tomando un liderazgo activo en el desarrollo de la pesquisa contra él.

Mientras este desarrollo explicaba, parcialmente, lo ocurrido en Quito aproximadamente entre 1736 y 1745, una perspectiva algo más amplia permitiría integrar en esta historia al tesorero Fernando García Aguado quien aparecía, en gran medida, como el primer responsable -en el campo local- del grupo y método luego adoptados por el presidente. García Aguado utilizaba su oficio con el fin de asegurar la elección de los suyos tanto al cabildo como para los cargos de alcaldes ordinarios. Era capaz, al parecer, de cohesionar un grupo que, respetando sus deseos, votaba según él le instruía. En cierto momento, el campo contrario, utilizando sus recursos económicos, lograba cambiar el balance de fuerzas y obtener algún control sobre el cabildo. Pero entonces, se volvió a recurrir a lo institucional, esta vez principalmente mediante la acción del presidente, quien gracias a su oficio era capaz de reconquistar el poder.

La lucha por controlar las instituciones -de carácter colectivo y no individual-incluía la formación de redes sociales. Esta formación influía en las instituciones a la vez que dependía de ellas. Según he intentado demostrar, el capital instituciones -encarnado en las facultades asociadas a diferentes oficiospodía llegar a ser fundamental en aquella lucha. Sin embargo, su victoria no se garantizaba cuando otros capitales entraban enjuego, por ejemplo, el dinero. Dentro del ámbito quiteño el papel institucional de los oficiales reales permitía un control sobre las elecciones al cabildo y en su seno. Durante la década de 1730, por lo menos, este hecho fue aprovechado continuamente por el tesorero Femando García Aguado quien pudo en consecuencia fortalecer un grupo social y garantizar la primacía de sus intereses 
tanto sociales como judiciales. Coincidiendo con el presidente, el tesorero pudo acumular un capital social e institucional que sus rivales sólo conseguían mediante un considerable gasto económico. La constitución de la red social de por sí, por lo tanto, no bastaba. Para que mera funcional y tuviera éxito, la misma necesitaba de acceso a diferentes recursos, económicos o institucionales. ${ }^{22}$

En un plano más general, la historia acaecida en Quito a mediados del siglo XVIII demuestra que las redes sociales podían tener diferentes lógicas tanto de asociación como de actuación. Mientras que algunas redes dependían de una sola persona (como era el caso de García Aguado), otras se basaban en alianzas mutuas entre varias (el grupo contrario); Mientras algunas se organizaban intencionalmente y con mucha pre-planificación (García Aguado), otras parecían más accidentales y espontáneas. Mientras el grupo de García Aguado se basaba en la familia y el clientelismo, el grupo contrario se cohesionaba alrededor de la familia y los intereses comerciales, pero algunos de sus miembros también se reclutaban casi al azar y todo lo que les ataba al grupo era una asociación temporal e instrumental. Los mecanismos de acción también variaban. Mientras un grupo, a raíz de la identidad de sus componentes (un tesorero real y eventualmente, el presidente de la audiencia) tenía acceso a capitales institucionales, los que no dudaba en utilizar, el otro sólo podía confiar en su capacidad económica a fin de manipular las instituciones. Dentro de la lógica del antiguo régimen, no es sorprendente que el segundo grupo, basado en el poder económico, acabara perdiendo terreno. Al fin y al cabo, dentro de una sociedad que daba importancia al poderío económico, pero que privilegiaba el honor, el status y la posición institucional, era evidente que el dinero no bastaba ni para controlar a las instituciones, ni para formar alianzas duraderas. El dinero podía traducirse en status y poderío local, pero para que esto ocurriera, hacía falta la intervención de las instituciones.

Otra conclusión importante, es la ausencia de actores individuales. Aunque cada una de las personas pertenecientes a los grupos enfrentados tenía cierta libertad a la hora de elegir su postura, la misma formación social, que dividía la ciudad en

${ }^{22}$ Con ello no se pretende decir que las redes se constituían sólo a raíz de la lucha por controlar las instituciones y que no tuvieran otras razones de ser. Simplemente, se quiere hacer constar que su éxito y su cohesión dependían también del acceso a las instituciones, es decir, a un cierto capital de alta importancia. 
dos bandos rivales, necesariamente limitaba el radio de actuación. A mediados del siglo XVIII, era evidente que uno debería pertenecer a una formación o a otra y que, esta pertenencia, significaba tanto derechos como deberes, tanto ventajas como desventajas. Aunque teóricamente posible, el movimiento de un grupo a otro apenas ocurría y, a medida que la lucha se intensificaba, incluso los que querían quedarse al margen de ella acababan identificados, si no enteramente insertos -incluso contra su voluntad- en una formación u otra. 